\title{
Construção compartilhada de material educativo sobre câncer de próstata
}

\author{
Renata Oliveira Maciel dos Santos, ${ }^{1}$ Danielle Nogueira Ramos ${ }^{1}$ \\ e Mônica de Assis ${ }^{1}$
}

Como citar

Santos ROM, Ramos DN, Assis A. Construção compartilhada de material educativo sobre câncer de próstata. Rev Panam Salud Publica. 2018;42:e122. https://doi.org/10.26633/RPSP.2018.122

RESUMO Objetivo. Apresentar o processo de construção compartilhada de material educativo sobre câncer de próstata.

Métodos. Foi desenvolvida uma pesquisa participante com uma amostra de conveniência que incluiu, inicialmente, 15 homens e uma mulher (média de idade: 52 anos), todos trabalhadores da mesma empresa, para subsidiar a elaboração de um material educativo. A primeira versão foi avaliada por oito especialistas (cinco especialistas em detecção precoce do câncer, um em urologia e dois em comunicação social) e um grupo diferente de 21 participantes (18 homens e três mulheres; média de idade: 41 anos). O material elaborado abordou riscos e benefícios do rastreamento do câncer de próstata.

Resultados. Os participantes desconheciam a orientação do Ministério da Saúde contra o rastreamento do câncer de próstata e houve debate sobre dúvidas e percepções relativas ao tema. O produto final, que gerou uma publicação do Instituto Nacional de Câncer José Alencar Gomes da Silva, foi avaliado por participantes e especialistas.

Conclusões. A participação dos homens na elaboração e na avaliação do material confirmou a necessidade imperiosa de difundir informações sobre riscos e potenciais danos associados ao rastreamento desse câncer. O material educativo não deve ser visto como peça isolada, mas como recurso complementar para ações educativas em saúde de caráter participativo, crítico e criativo.

Palavras-chave Neoplasias da próstata; comunicação em saúde; educação em saúde; decisão compartilhada.

Excetuando-se os cânceres de pele não melanoma, o câncer de próstata é o mais incidente em homens no Brasil. Um aumento nas taxas de incidência e mortalidade por esse câncer vem ocorrendo possivelmente pelo envelhecimento

\footnotetext{
Instituto Nacional de Câncer José de Alencar Gomes da Silva, Divisão de Detecção Precoce e Apoio à Organização de Rede, Rio de Janeiro (RJ), Brasil. Correspondência: Renata Oliveira Maciel dos Santos, renata.santos@inca.gov.br
}

populacional, advento de exames diagnósticos, melhoria nos sistemas de informação e aumento do rastreamento desse câncer (1).

O rastreamento do câncer de próstata tem sido extensamente debatido. Rastreamento é uma estratégia de detecção precoce que se caracteriza pela aplicação de exames periódicos em uma população assintomática, em determinada faixa etária, com objetivo de identificar uma dada doença precocemente (2). Estudos apontam ausência de evidências científicas para recomendar o rastreamento populacional do câncer de próstata, uma vez que o benefício é discreto ou ausente e acompanhado de potenciais danos à saúde dos homens (3-5).

O benefício esperado do rastreamento é identificar o câncer de próstata no seu início, a fim de impedir a sua progressão e aumentar as chances de tratamento. Os riscos são resultados falso-positivos, que provocam ansiedade e necessidade 
de novos exames, como biópsia, cujas complicações são dor, sangramento e infecções; resultados falso-negativos, que podem induzir à falsa segurança do homem e atrasar o diagnóstico; ou sobrediagnóstico e sobretratamento, que se referem ao diagnóstico e tratamento de um câncer que não evoluiria clinicamente e não ameaçaria a vida. Nesse caso, as complicações incluem incontinência urinária e disfunção sexual erétil, além de pequeno risco de morte (4-6).

O Ministério da Saúde do Brasil, assim como instituições internacionais, como as forças-tarefa americana e canadense, e países como Austrália e Reino Unido não recomendam a implantação de programas de rastreamento para o câncer de próstata. Ressaltam que a decisão deve ser compartilhada entre o médico e o paciente, depois de o paciente ter sido informado sobre os riscos e benefícios do rastreamento (6-8). Nesse processo, os envolvidos compartilham as melhores evidências disponíveis, discutem valores e preferências e os incluem na tomada de decisão sobre uma determinada ação em saúde (9).

Na contramão do debate sobre limites do rastreamento, cresce no Brasil a campanha do Novembro Azul, estimulada por sociedades médicas e organizações não governamentais, que incentiva os exames periódicos para detectar precocemente o câncer de próstata, apresentando equívocos e lacunas que limitam o direito dos homens de fazerem escolhas informadas sobre sua saúde (10).

Para informar melhor sobre o câncer de próstata e os riscos e benefícios do rastreamento, tema ausente no cenário da campanha, surgiu o desafio de criar um material educativo com a participação de homens, numa lógica dialógica e menos vertical de elaboração. A necessidade de informar a população sobre prevenção e detecção precoce do câncer é prevista na atual Política Nacional de Prevenção e Controle do Câncer (11) e em outras políticas e planos relacionados ao tema no Brasil (12, 13).

Muito se tem questionado sobre a forma linear que orienta a produção de materiais educativos em saúde. Simplificações, generalizações e reducionismos, com predomínio do ponto de vista técnico, são alguns dos problemas apontados (14-16).

Na busca de rever esse padrão, autores destacam a importância da participação na construção e avaliação de materiais educativos, defendendo a produção compartilhada como oportunidade para o maior envolvimento dos sujeitos com as informações apresentadas (17-19). A correspondência entre o conteúdo abordado e os interesses e necessidades da população é um cuidado a ser buscado nessa construção, que deve reconhecer o contexto sociocultural e político em que as pessoas estão inseridas $(18,20)$.

Esse caminho é ainda pouco percorrido e integra a agenda do campo da comunicação e saúde no Brasil, conforme diretriz aprovada na Conferência Nacional de Saúde, de 2015 (21): "fortalecer o SUS como política pública, assegurando a democratização do acesso à informação em saúde, facilitando a interação entre trabalhadoras(es), usuárias(os) e gestoras(es) nos níveis municipais, estaduais e federal, promovendo a educação na perspectiva da construção coletiva e dialógica" (p. 7). A construção compartilhada alinha-se à perspectiva da Educação Popular em Saúde, que propõe o diálogo e o encontro de saberes na relação entre profissionais e população, sendo hoje referência para as práticas educativas no Sistema Único de Saúde (SUS) (22).

Nesse contexto, o objetivo do presente artigo é apresentar o processo de construção compartilhada de material educativo sobre câncer de próstata desenvolvido por uma instituição federal especializada em atenção oncológica e responsável por desenvolver ações nacionais de controle do câncer.

\section{MATERIAL E MÉTODOS}

A construção do material educativo foi definida como pesquisa participante, no sentido de uma ação coletiva na qual os pesquisadores e os sujeitos envolvidos na situação interagem de forma cooperativa e participativa, buscando responder a uma questão proposta (23). A questão geral do trabalho foi: como deve ser um material educativo sobre câncer de próstata? Essa pergunta foi trabalhada em grupo com 15 homens e uma mulher e subsidiou a elaboração do material, que foi avaliado por oito especialistas e 21 participantes diferentes do grupo inicial. As etapas do processo são descritas a seguir.

\section{Levantamento bibliográfico}

Foi realizada na Internet a busca livre de materiais educativos para a população sobre detecção precoce do câncer de próstata, produzidos por instituições nacionais e internacionais. A busca se deu no browser Google, com as expressões rastreamento do câncer de próstata e detecção precoce do câncer de próstata. Foram também pesquisados materiais nos sites eletrônicos das principais instituições que trabalham com o tema, como sociedades médicas e instituições governamentais de outros países, com o filtro de língua portuguesa, inglesa e espanhola, sem limitação de ano. Ao total foram encontrados 60 materiais educativos, a maioria publicações internacionais de países como Estados Unidos, Canadá e Reino Unido.

Essa busca subsidiou a organização dos tópicos a serem discutidos na atividade educativa com o grupo de homens e a elaboração do conteúdo técnico do material educativo.

\section{Atividade educativa}

A atividade educativa foi realizada em formato de roda de conversa, com um grupo de homens, funcionários de uma empresa de informática no Centro do Rio de Janeiro. A escolha desse cenário se deu após a empresa solicitar à instituição pesquisadora a presença de um profissional para realizar uma atividade educativa sobre câncer de próstata. Essa empresa possui um número significativo de trabalhadores do sexo masculino, na faixa etária de interesse para o estudo, de 40 a 60 anos, além de estar localizada próxima à instituição. A participação dos homens foi voluntária e a escolha dessa amostra foi por conveniência, considerando a concentração de homens nesse local.

No início da atividade, foi apresentada a proposta de produzir um material educativo a partir das dúvidas e questionamentos dos participantes sobre o tema, sendo esses incentivados a participarem ativamente. A atividade educativa durou 3 horas, foi coordenada por dois enfermeiros e buscou captar os principais questionamentos dos homens sobre o câncer de próstata e definir a linguagem utilizada para expressar as dúvidas, o tipo de ilustração a ser usado e o melhor formato para o material.

\section{Análise dos dados e produção do material educativo}

Os dados obtidos na roda de conversa (áudio e impressões do observador) 
foram analisados, agrupados e categorizados. Esses dados subsidiaram a elaboração de um conteúdo preliminar que foi submetido à avaliação de especialistas das áreas parceiras na produção do material: cinco especialistas em detecção precoce do câncer, um especialista em urologia e dois em comunicação social. Depois das contribuições, o conteúdo final foi enviado para programação visual com as sugestões de ilustração e formatação.

\section{Avaliação do material educativo}

O material produzido foi submetido à avaliação de dois grupos de homens em dois momentos: por formulário eletrônico e presencialmente. Inicialmente, foi proposto um encontro presencial com o mesmo grupo que participou da atividade educativa, porém não foi possível a participação desses homens no horário disponível e optou-se pelo envio de um formulário eletrônico com questões fechadas relacionadas ao conteúdo, formatação, ilustrações e linguagem. O formulário foi enviado por e-mail juntamente com o material produzido.

A dificuldade do reencontro presencial com o mesmo grupo e a baixa taxa de resposta ao formulário eletrônico foram contornadas com a realização de um segundo momento de avaliação. Desse segundo momento participou um novo grupo de homens, trabalhadores da mesma empresa, que compareceram voluntariamente, após convite para uma atividade educativa sobre câncer de próstata.

A atividade teve início com a leitura conjunta do material projetado em tela, seguida de análise individual do conteúdo e formato do material impresso. Foi utilizado o mesmo roteiro de avaliação do formulário eletrônico para a discussão do grupo.

As contribuições obtidas foram analisadas e subsidiaram a reformulação do material. A programação visual e o texto foram ajustados para contemplar, tanto quanto possível, as sugestões dos envolvidos no processo.

Previamente ao início das atividades, os participantes foram informados sobre os objetivos da pesquisa, a dinâmica a ser seguida, o caráter voluntário de sua participação e o registro da atividade por dois gravadores de áudio. A seguir, o termo de consentimento livre e esclarecido
(TCLE) foi lido e assinado. A gravação foi transcrita e analisada pelos pesquisadores, sendo mantida em sigilo a identidade dos participantes. $\mathrm{Na}$ avaliação eletrônica, não foi necessária a identificação dos participantes.

O protocolo de estudo foi aprovado pelo Comitê de Ética em Pesquisa da instituição pesquisadora (CAAE: 52822816.0.0000.5274).

\section{RESULTADOS}

A atividade educativa com o primeiro grupo contou com 15 homens e uma mulher, na faixa etária de 19 a 60 anos. A média de idade foi de 52 anos. Dos participantes, 10 eram casados, 11 tinham escolaridade superior, quatro tinham escolaridade média e 13 eram usuários de planos de saúde. Durante a atividade, a interação dos participantes foi descontraída e houve muita participação, o que foi facilitado pelo fato de todos partilharem o mesmo ambiente de trabalho.

Os questionamentos do grupo estão apresentados segundo conteúdo na tabela 1. As dúvidas sobre tratamento e prognóstico do câncer de próstata foram respondidas, porém não incorporadas ao material educativo em função do seu escopo ser a detecção precoce e a prevenção.

Todos os participantes desconheciam a orientação do Ministério da Saúde de não recomendar o rastreamento do câncer de próstata e os possíveis riscos relacionados à realização desses exames:

o que me ensinaram é que eu tenho que fazer exames de rotina todos os anos. (H1)

Os homens tinham informação de que era preciso fazer check-up anual e ficaram surpresos pelo fato de seus médicos pedirem esses exames, sem informar sobre os riscos. Alguns ficaram intrigados com os possíveis danos dos exames de rastreamento e tiveram dificuldade de entender os riscos da realização de exames não invasivos como rotina. Foi explicado que a coleta de sangue para o antígeno prostático específico (PSA) e o toque retal apresentam baixo risco durante sua execução e que os riscos se relacionam a resultados incorretos, sobrediagnóstico e sobretratamento.

TABELA 1. Dúvidas apresentadas por homens participantes de atividade educativa para construção de material informativo sobre câncer de próstata, Rio de Janeiro, Brasil, 2017

\begin{tabular}{|c|c|}
\hline Tópico & Dúvida \\
\hline $\begin{array}{l}\text { Fisiologia e anatomia } \\
\text { da próstata }\end{array}$ & $\begin{array}{l}\text { - } 0 \text { tamanho da próstata aumenta com a idade? } \\
\text { - Todos os homens terão câncer de próstata? }\end{array}$ \\
\hline $\begin{array}{l}\text { Causas e fatores de } \\
\text { risco }\end{array}$ & $\begin{array}{l}\text { - Quais as causas e fatores de riscos para o câncer de próstata? } \\
\text { - Qual é o grupo de risco para ter câncer de próstata? } \\
\text { - Existe idade mínima para ter câncer de próstata? } \\
\text { - Qual idade possui maior incidência? } \\
\text { - O histórico familiar influencia o desenvolvimento do câncer de próstata? } \\
\text { - Fatores ambientais influenciam o desenvolvimento do câncer de próstata? } \\
\text { - Tabagismo, alimentação e forma de defecar estão associados ao câncer de próstata? } \\
\text { - Ficar sentado muito tempo aumenta o risco de ter câncer de próstata? }\end{array}$ \\
\hline Prevenção & - Como se previne o câncer de próstata? \\
\hline Sinais e sintomas & $\begin{array}{l}\text { - Quais são os sinais e sintomas da doença? } \\
\text { - Como identificar o câncer de próstata? }\end{array}$ \\
\hline Métodos diagnósticos & $\begin{array}{l}\text { - Que fatores alteram o exame de antígeno prostático específico (PSA) e quais são as } \\
\text { recomendações para a realização do exame? } \\
\text { - Como é feito o exame de toque retal? } \\
\text { - Qual é a limitação do toque retal? } \\
\text { - Existem métodos não invasivos para detectar precocemente o câncer de próstata? } \\
\text { - Qual é o exame mais indicado para cada idade? } \\
\text { - A ultrassonografia é utilizada para diagnosticar precocemente o câncer de próstata? }\end{array}$ \\
\hline $\begin{array}{l}\text { Recomendação para o } \\
\text { rastreamento }\end{array}$ & $\begin{array}{l}\text { - É preciso fazer algum exame de rotina? } \\
\text { - Qual é a periodicidade recomendada para realizar exames relacionados ao câncer de } \\
\text { próstata? } \\
\text { - Homens com mais de } 40 \text { anos devem fazer o teste PSA? } \\
\text { - Qual é a idade recomendada para iniciar a realização dos exames? } \\
\text { - Há mudança de recomendação de acordo com a faixa etária? }\end{array}$ \\
\hline $\begin{array}{l}\text { Prognóstico e } \\
\text { tratamento }\end{array}$ & $\begin{array}{l}\text { - Qual o prognóstico do câncer de próstata, quando comparado com outros tipos de } \\
\text { cânceres? } \\
\text { - Dependendo do estadiamento do câncer, qual a abordagem para o tratamento? }\end{array}$ \\
\hline
\end{tabular}


Se você já está ali qual é o problema em fazer o PSA? (H4)

Meu médico nunca me falou isso. (H6)

A utilização de outros métodos, como a ultrassonografia, também foi mencionada:

Se eu faço uma ultrassonografia e já verifico que a próstata está num tamanho considerado padrão, normal, eu deveria fazer alguma coisa a mais nesse momento? Não. Então significa para mim que o exame não é "invasivo", o fato de já me mostrar que o tamanho está normal, já me isenta de qualquer tipo de preocupação. (H1)

As perguntas do grupo foram respondidas à medida que os tópicos eram abordados. Houve debate sobre a falta de acurácia da ultrassonografia para rastrear o câncer de próstata. Outros questionamentos foram:

Os sintomas [de câncer de próstata] são parecidos com crise renal e doenças benignas da próstata? (H10)

Qual é a diferença entre exames de investigação e exames de confirmação diagnóstica? (H12)

Ao final da atividade, foi perguntado ao grupo qual o melhor formato para o material educativo - folder ou cartilha - e houve consenso quanto à cartilha, pois "são muitas informações relevantes e em um folder isso não caberia". Imagens da próstata também foram apresentadas para que os participantes escolhessem a mais apropriada. As dúvidas, percepções e comentários embasaram a elaboração da cartilha, que foi organizada no formato de perguntas e respostas sobre os pontos de interesse e ilustrada para facilitar a compreensão e tornar o material mais atraente.

Esse material foi posteriormente enviado para avaliação por especialistas e recebeu as seguintes contribuições: sobre a detecção precoce do câncer, os especialistas sugeriram acrescentar na cartilha informações sobre o papel da próstata na sexualidade do homem; inserir uma seção de apresentação para aproximar o leitor do material e apresentar, em destaque, a recomendação do Ministério da Saúde (figura 1). Sobre questões de urologia, os especialistas sugeriram inserir a pergunta "qual exame confirma o câncer de próstata?"; e sobre aspectos de comunicação, as sugestões foram inserir um texto explicando o processo de formação de câncer, colocar a recomendação do Ministério da Saúde ao final da cartilha, seguida da mensagem sobre decisão compartilhada, e substituir alguns termos para maior compreensão do conteúdo.

A avaliação pelos homens que participaram do primeiro grupo teve por finalidade captar suas impressões sobre a adequação do material quanto ao conteúdo e à forma. Obteve-se retorno do

FIGURA 1. Recomendação sobre o rastreamento do câncer de próstata no material educativo elaborado por meio de construção compartilhada, Rio de Janeiro, 2017
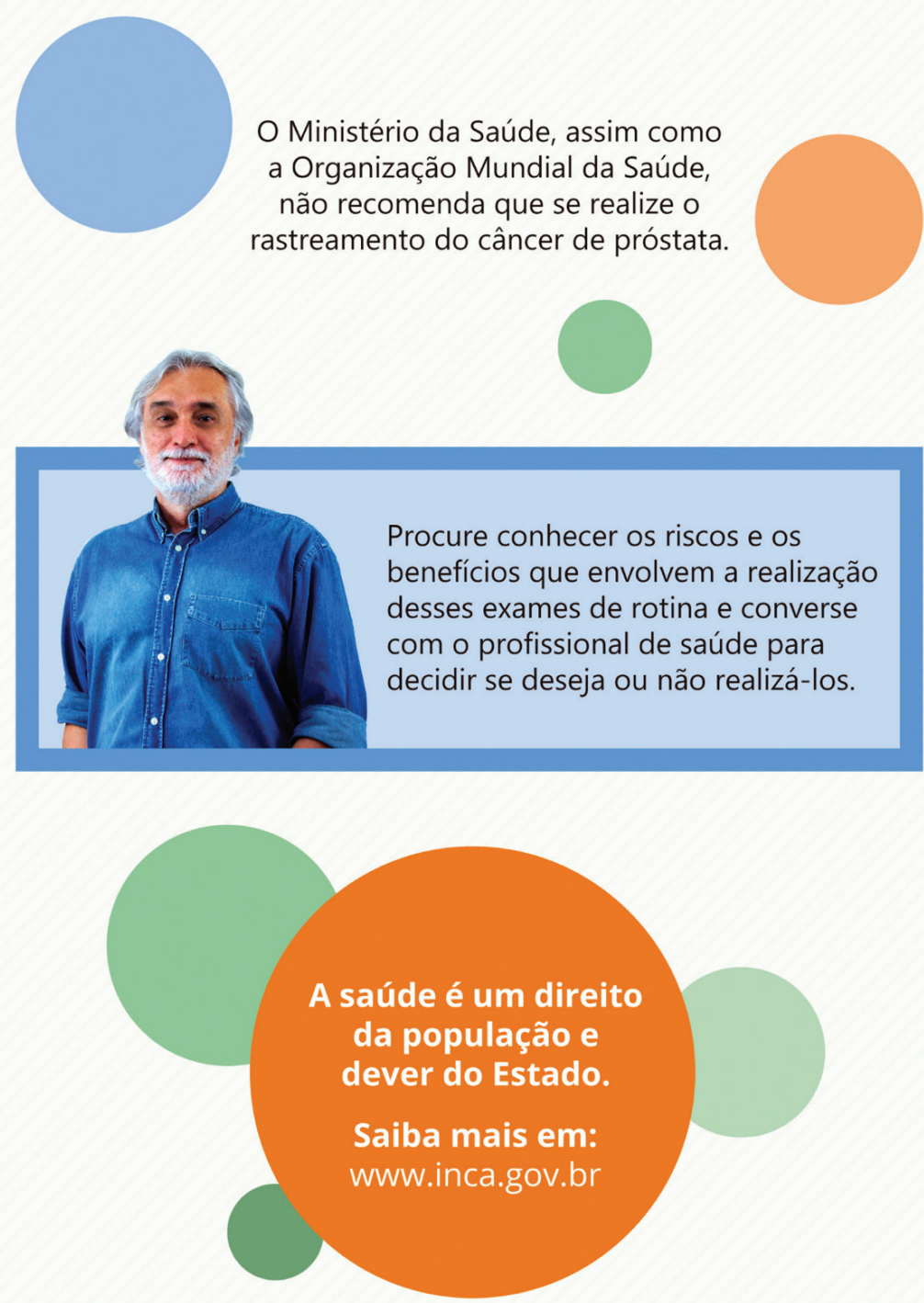

12

Câncer de próstata: Vamos falar sobre isso?

Fonte: INCA. A versão completa da cartilha está disponível no Apêndice. 
todos consideraram a quantidade de texto satisfatória.

$\mathrm{Na}$ avaliação presencial, estiveram presentes 18 homens e três mulheres, na faixa etária de 25 a 62 anos, com média de idade de 41 anos. Nove eram casados, 16 tinham acesso ao sistema de saúde privado, 12 tinham escolaridade superior e nove tinham escolaridade média. A participação dos homens foi bem ativa na avaliação, sugerindo modificações nas ilustrações e na diagramação do conteúdo. A presença de mulheres não foi planejada, porém não houve objeção a sua permanência, pois o material educativo proposto, embora direcionado prioritariamente aos homens, destina-se a toda a população. Durante a atividade não foram registradas contribuições específicas das mulheres na discussão do material.

O grupo avaliou o conteúdo como adequado e explicativo, não identificando termos de difícil compreensão. As observações levantadas pelo grupo foram:

Ter um título mais provocativo, trazendo a temática dos riscos e benefícios. (H17)

Modificar a ilustração relacionada ao peso adequado, pois o ícone não estava claro. (H15)

Organizar a recomendação sobre a detecção precoce do câncer de próstata em um fluxograma. (H20)

As contribuições foram analisadas e parcialmente incorporadas na versão final da cartilha, considerando as opiniões dos especialistas e a viabilidade técnica das mudanças no leiaute. O produto final está disponível como Apêndice.

\section{DISCUSSÃO}

O presente artigo descreveu a construção de material educativo sobre câncer de próstata com participação do público-alvo das informações compiladas. As questões e tópicos de interesse levantados pelo grupo de homens participantes foram, em parte, similares ao conteúdo abordado nos materiais educativos internacionais pesquisados, exceto quanto aos riscos e benefícios do rastreamento do câncer de próstata. O distanciamento dos participantes quanto a esse aspecto provavelmente reflete a pequena discussão sobre o tema no contexto brasileiro, confirmada nos materiais educativos nacionais encontrados. Essa lacuna vem sendo apontada por autores que alertam para o viés que orienta campanhas como o Novembro Azul, destacando a necessidade de se olhar com cuidado os interesses envolvidos (24-26).

Nos materiais educativos de outros países, identificados na busca, além de aspectos gerais sobre câncer de próstata, observou-se a valorização da decisão compartilhada e a apresentação dos riscos e benefícios do rastreamento. Esse aspecto não foi tratado nos materiais nacionais. Apenas dois mencionaram controvérsias, mas não abordaram os benefícios e riscos do rastreamento. De fato, no presente estudo, observou-se o desconhecimento dos participantes sobre os riscos envolvidos no rastreamento do câncer de próstata, indicando a pequena valorização da participação dos homens nas decisões sobre sua saúde negligenciando-se assim o direito à autonomia, como recomendado por protocolos nacionais e internacionais. É ainda distante da prática o imperativo ético de participação dos indivíduos no processo de tomada de decisão, principalmente nas intervenções que possuem alto grau de incerteza sobre seu benefício líquido, como no caso do rastreamento do câncer de próstata (27).

A discussão sobre o balanço dos riscos e benefícios das intervenções médicas em saúde é incipiente na prática médica no Brasil. Grande parte dos profissionais de saúde e da população acredita que a detecção precoce de doenças em estágio assintomático é uma ação livre de riscos e desconhece as evidências que embasam a decisão de não recomendar o rastreamento. Por essa razão, cresce a preocupação com a prevenção quaternária, dimensão do cuidado que busca reduzir os riscos de intervenções médicas e sanitárias (28).

Em pesquisa com médicos da atenção primária, Colão (29) identificou, além disso, medo e indisposição para discutir o rastreamento do câncer de próstata com homens que trazem a demanda de check-up. Alguns médicos argumentam que muito tempo é gasto com explicações sobre os motivos da não indicação do exame de PSA, sendo que muitas vezes essas explicações não são compreendidas pelo paciente. Outros médicos têm informação sobre o protocolo, porém prescrevem o exame em função de suas percepções afetivas e sociais, sem compartilhar informações com os homens. Embora estudos internacionais relacionem a decisão compartilhada a melhores resultados, aumento da satisfação e da percepção de risco, proporcionando escolhas com mais qualidade, a prática de envolver os usuários em decisões sobre saúde é raramente implementada nos cenários de cuidados $(30,31)$.

Apesar da brevidade da atividade educativa realizada neste estudo, a boa receptividade e o entrosamento prévio dos participantes favoreceram a dinâmica participativa nos grupos. A realização do estudo no cenário do ambiente de trabalho parece ter sido uma estratégia capaz de agregar os homens, segmento que frequenta menos os espaços de cuidado à saúde, em torno dessa discussão (32).

A avaliação do material por outro grupo, além daquele que trabalhou na produção, apesar de não prevista no primeiro momento, permitiu um novo olhar e trouxe contribuições interessantes. $\mathrm{Na}$ experiência de Mialhe (19), a elaboração de materiais educativos com usuários de uma unidade básica, em sala de espera, permitiu o levantamento de interesses e a aproximação com a linguagem dos usuários, sendo os materiais elaborados também avaliados por outros participantes usuários da clínica.

A avaliação dos especialistas demandou um esforço de mediação para enriquecer o material com a visão interdisciplinar que o tema demanda. O rastreamento do câncer de próstata apresenta divergências e polêmicas e foi preciso buscar o diálogo para negociar as visões e chegar a um conteúdo equilibrado e coerente, agregando as contribuições de todos os parceiros.

A limitação identificada na pesquisa foi a impossibilidade de envolver um maior número de homens, com perfis socioeconômicos distintos, na produção e avaliação do material, como previsto inicialmente. Em consequência, o trabalho ficou restrito a homens com bom nível de escolaridade e renda, o que pode ter facilitado a compreensão do material educativo. Ao considerar, porém, que indivíduos com maior acesso aos exames de rastreamento estão mais expostos aos possíveis riscos dessa prática, constata-se a relevância das informações contidas no material educativo, cujo público-alvo é extenso e não se resume à população com menor acesso à informação e aos serviços de saúde. 
Cabe reconhecer a necessidade de produção descentralizada de material educativo sobre câncer e demais temas de saúde, considerando a diversidade do país e o uso de diferentes mídias. Isso não apenas pelas desigualdades no acesso e na compreensão de informações, mas também pelas particularidades socioculturais a serem consideradas nos recursos de comunicação em saúde. Apesar de ser uma prática recomendada, a descentralização encontra ainda limites na insuficiência de estruturação de grande parte das secretarias de saúde do Brasil (33).

A condição de curta duração da pesquisa em um ambiente de trabalho limitou, provavelmente, a emergência de outros assuntos relacionados ao câncer de próstata, como, por exemplo, a sexualidade (24). A proposta viabilizou abordar dúvidas e anseios sobre o tema, mas não foi possível avançar para a produção de um texto mais inovador em termos de saúde do homem, que ampliasse o foco além da doença.

O diálogo intersetorial envolvendo três áreas técnicas de uma mesma instituição também envolveu dificuldades e limites e impôs desafios ao papel mediador exercido pela equipe de Gomes da Silva (INCA). Estimativa 2018: incidência de câncer no Brasil. Rio de Janeiro: INCA; 2018. Disponível em http: / / www.inca.gov.br/estimativa/2018/estimativa-2018.pdf Acessado em 23 de abril de 2018.

2. World Health Organization (WHO). WHO cancer control: knowledge into action: WHO guide for effective programmes; module 3. 2007. Disponível em: http:// www.who.int/cancer/modules/en / Acessado em junho de 2018.

3. Andriole GL, Crawford ED, Grubb RL 3rd, Buys SS, Chia D, Church TR, et al. Prostate Cancer Screening in the Randomized Prostate, Lung, Colorectal, and Ovarian Cancer Screening Trial: Mortality results after 13 years of follow-up. J Natl Cancer Inst. 2012;104(2):125-32.

4. Instituto Nacional de Câncer José Alencar Gomes da Silva (INCA). Rastreamento do câncer de próstata. Rio de Janeiro: INCA; 2013. Disponível em: http://www1.inca. gov.br/inca / Arquivos/rastreamento_ prostata_resumido.2013.pdf Acessado em 13 de novembro de 2017.

5. Martin RM, Donovan JL, Turner EL, Metcalfe C, Young GJ, Walsh EI, et al. Effect of a low-intensity PSA-based screening intervention on prostate cancer mortality: the CAP Randomized Clinical Trial. pesquisa na condução do processo. Ao mesmo tempo, porém, promoveu um exercício de integração institucional na busca de articulação do processo de trabalho.

A experiência relatada neste artigo reconhece a complexidade envolvida na produção de sentidos e buscou experimentar processos participativos de construção. Destaca-se que o material educativo não deve ser entendido como peça isolada, que ocupa o vazio de relações entre profissionais de saúde e usuários, mas sim como recurso complementar, apoio de ações educativas de caráter participativo, crítico e criativo, a serem promovidas no contexto da saúde e em outros espaços sociais.

$\mathrm{O}$ estudo atendeu a proposta de produzir um material educativo a partir de uma perspectiva dialógica, ao ouvir um grupo de homens sobre seus interesses e opiniões sobre o câncer de próstata. A participação dos homens na elaboração e na avaliação do material confirmou a necessidade imperiosa de difundir informações sobre riscos e possíveis danos associados ao rastreamento desse câncer. A cartilha está disponível em versões online e impressa. O processo de produção e avaliação não

\section{REFERÊNCIAS}

JAMA. 2018; 19(9):883-95. Disponível em: http:/ / doi:10.1001/jama.2018.0154 Acessado em 27 de abril de 2018.

6. Schröder FH, Hugosson J, Roobol MJ, Tammela TLJ, Zappa M, Nelen V, et al. The European Randomized Study of Screening for Prostate Cancer - prostate cancer mortality at 13 years of follow-up. Lancet. 2014;384(9959):2027-35.

7. Barret B, McKenna P. Communicating benefits and risks of screening for prostate, colon, and breast cancer. Fam Med. 2011;43(4):248-53.

8. Ministério da Saúde, Instituto Nacional de Câncer (INCA). Nota técnica conjunta $\mathrm{n}^{\circ}$ 001/2015. Posicionamento do Ministério da Saúde acerca da integralidade da saúde dos homens no contexto do Novembro Azul. Brasil 2015. Disponível em: http:// www1.inca.gov.br/inca/Arquivos/nota tecnica_saude_do_homem_ms.pdf Acessado em 13 de nov de 2017.

9. Scholl I, LaRussa A, Hahlweg P, Kobrin S, Elwyn G. Organizational- and systemlevel characteristics that influence implementation of shared decision-making and strategies to address them - a scoping review. Implement Sci. 2018;13(1):40.

10. Sociedade Brasileira de Medicina de Família e Comunidade. Comunicado aos (às) apoiadores(as) da campanha Novembro Azul. Rio de Janeiro, outubro se encerrou em si mesmo e deverá ser qualificado em estudos de recepção que avaliem o material junto a outros grupos de homens, de menor nível de escolaridade e renda, a fim de verificar a comunicabilidade do material e as questões daí emergentes.

Espera-se que a experiência aqui apresentada estimule a construção de materiais educativos em diversos formatos $\mathrm{e}$ cenários, baseados em uma metodologia participativa, que possa acolher demandas da saúde do homem de forma mais inclusiva e dialógica.

Agradecimentos. Agradecemos à equipe da Divisão de Detecção Precoce do INCA/MS, especialmente a Marcos André Félix da Silva, pelas contribuições no processo de produção do material educativo aqui apresentado.

Conflitos de interesse. Nada declarado pelos autores.

Declaração. As opiniões expressas no manuscrito são de responsabilidade exclusiva dos autores e não refletem necessariamente a opinião ou políticas da RPSP/PAJPH ou da Organização Pan-Americana da Saúde (OPAS). de 2015. Disponível em: http://www. sbmfc.org.br/media/Novembro\%20Azul. pdf Acessado em 13 de novembro de 2017.

11. Brasil. Portaria MS/GM 874/2013. Disponível em: http://bvsms.saude.gov. $\mathrm{br} / \mathrm{bvs} /$ saudelegis/gm/2013/prt0874 16_05_2013.html Acessado em junho de 2018

12. Brasil, Ministério da Saúde. Plano de Ações Estratégicas para o enfrentamento das doenças crônicas não transmissíveis (DCNT) no Brasil - 2011-2022. Brasília: Ministério da Saúde; 2011. (Série B. Textos Básicos de Saúde). Disponível em: http:// bvsms.saude.gov.br/bvs/publicacoes/ plano_acoes_enfrent_dcnt_2011.pdf Acessado em junho de 2018.

13. Brasil, Ministério da Saúde. Portaria MS/ GM 2 446/2014. Disponível em: http:// bvsms.saude.gov.br/bvs/saudelegis/ gm/2014/prt2446_11_11_2014.html Acessado em junho de 2018

14. Freitas FV, Rezende Filho LA. Modelos de comunicação e uso de impressos na educação em saúde: uma pesquisa bibliográfica. Interface (Botucatu). 2011;15(36): 243-56.

15. Vasconcelos PR. Próteses de comunicação e alinhamento comportamental sobre impressos. Rev Saude Publica. 2003;37(4): 531-42.

16. Vasconcelos EM. Educação popular: de uma prática alternativa a uma estratégia 
de gestão participativa das políticas de saúde. Physis. 2004;14(1):67-83.

17. Souza K, Rozemberg B, Kelly-Santos A, Yasuda N, Sharapin M. O desenvolvimento compartilhado de impressos como estratégia de educação em saúde junto a trabalhadores de escolas da rede pública do Estado do Rio de Janeiro. Cad Saude Publica. 2003;19(2):495-504.

18. Carvalho MAP. A construção compartilhada do conhecimento: análise da produção de material educativo. Em: Brasil. Ministério da Saúde. Caderno de Educação Popular e Saúde. Brasília: Ministério da Saúde; 2007. Pp. 91-101. (Série B. Textos Básicos de Saúde).

19. Mialhe FL. Dialogando com o saber popular na elaboração de materiais educativos. Em: Mano MAM, Prado EV, organizadores. Vivências de educação popular na atenção primária à saúde: a realidade e a utopia. São Carlos: Edufscar; 2010. Pp. 193-8.

20. Reberte LM, Hoga LAK, Gomes ALZ. O processo de construção de material educativo para a promoção da saúde da gestante. Rev Latino-Am Enfermagem. 2012;20(1):101-8.

21. Conselho Nacional de Saúde. $15^{\text {a }}$ Conferência Nacional de Saúde. Diretrizes aprovadas nos Grupos de Trabalho ou na Plenária Final. Disponível em: https:// www.abrasco.org.br/site/wp-content/ uploads / 2016/01/Relat\%C3\%B3rio_ Diretrizes_15CNS.pdf Acessado em 16 de novembro de 2017

22. Brasil, Ministério da Saúde. Portaria MS/ GM 2 761/2013. Disponível em: http:// bvsms.saude.gov.br/bvs/saudelegis / gm/2013/prt2761_19_11_2013.html Acessado em junho de 2018.

23. Thiollent M. Pesquisa-ação nas organizações. São Paulo: Atlas; 1997.

24. Modesto A, Lima RLB, D'Angelis AC, Augusto DK. Um novembro não tão azul: debatendo rastreamento de câncer de próstata e saúde do homem. Interface (Botucatu). 2018;22(64):251-62.

25. Assis M. Campanha demais e informação de menos. Interface (Botucatu). 2018; 22(64):269-71.

26. Gérvas J. ¿Noviembre azul? En ningún caso. Interface. 2018; 22(64):267-8.

27. Elwyn G, Cochran N, Pignone M. Shared decision making - the importance of diagnosing preferences. JAMA Intern Med. 2017;177(9):1239-40.

28. Norman AH, Tesser CD. Prevenção quaternária na atenção primária à saúde: uma necessidade do Sistema Único de Saúde. Cad Saude Publica. 2009;25(9):2012-20.

29. Colão CF. Saúde do homem Conhecimento sobre o rastreamento do câncer de próstata por profissionais da Estratégia de Saúde da Família na Zona Oeste do Rio de Janeiro. [dissertação de mestrado em Saúde Pública]. Rio de Janeiro: Escola Nacional de Saúde Pública Sérgio Arouca; 2015.

30. Härter M, Moumjid N, Cornuz J, Elwyn G, van der Weijden T. Shared decision making in 2017: international accomplishments in policy, research and implementation. Z Evid Fortbild Qual Gesundhwes. 2017;123-124:1-5

31. Cochran N, Pignone M Elwyn G. Shared decision making - the importance of diagnosing preferences. JAMA Intern Med. 2017;177(9):1239-40.

32. Silva LAV, Santos M, Dourado I. Entre idas e vindas: histórias de homens sobre seus itinerários ao serviço de saúde para diagnóstico e tratamento de HIV / Aids. Physis. 2015;25(3):951-73.

33. Araujo IS, Cardoso JM. Comunicação e saúde. Rio de Janeiro: Fiocruz; 2007.

Manuscrito recebido em 29 de agosto de 2017. Aceito em versão revisada em 27 de abril de 2018.
ABSTRACT

\section{Shared development of prostate cancer education material}

Objective. To describe the process of shared development of a prostate cancer education booklet.

Methods. A participant observation study was performed with a convenience sample which initially included a group of 16 co-workers (15 men and one woman) to gather information for the development of educational material. The first draft was assessed by eight specialists (five specialists in early cancer detection, one specialist in urology, and two communication specialists) and by a different group of 21 co-workers from the same company (18 men and three women). The booklet addressed the risks and benefits of prostate cancer screening.

Results. The participants were not aware of the Brazilian Health Ministry recommendation against prostate cancer screening. The group discussed their doubts and perceptions regarding the theme. The final booklet, which became a publication by the José Alencar Gomes da Silva National Cancer Institute (INCA), was evaluated by participants and specialists.

Conclusions. The participation of men in the development and assessment of the material confirmed the pressing need to disseminate information regarding the risks and potential damage associated with prostate cancer screening. Educational materials should not be treated as independent items, but rather as complementary resources to be used as part of shared, critical, and creative educational initiatives.

Keywords Prostatic neoplasms; health communication; health education; decision making. 
RESUMEN Objetivo. Describir el proceso de elaborar material educativo sobre el cáncer de próstata de manera compartida.

Métodos. Se realizó una investigación participativa con una muestra de conveniencia constituida, inicialmente, por un grupo de 15 hombres y una mujer (con una media

Elaboración compartida de material educativo sobre el cáncer de próstata de edad de 52 años), todos trabajadores de la misma empresa, con el fin de contribuir a la elaboración de material educativo. La evaluación de la primera versión estuvo a cargo de ocho especialistas (cinco en detección temprana del cáncer, uno en urología y dos en comunicación social) y de otro grupo de 21 participantes (18 hombres y tres mujeres con una media de edad de 41 años). En el material elaborado se abordaron los riesgos y beneficios del tamizaje del cáncer de próstata.

Resultados. Los participantes desconocían la recomendación dada por el Ministerio de Salud del Brasil contra el tamizaje del cáncer de próstata y hubo debate sobre dudas y percepciones relativas al tema. Los participantes y los especialistas evaluaron el producto final, que se convirtió en una publicación del Instituto Nacional del Cáncer José Alencar Gomes da Silva.

Conclusiones. La participación de los hombres en la elaboración y evaluación del material confirmó la imperiosa necesidad de difundir información sobre los riesgos y posibles perjuicios relacionados con el tamizaje de ese tipo de cáncer. El material educativo no debe verse como una obra aislada, sino como un recurso complementario para actividades educativas en salud de carácter participativo, crítico y creativo.

Palabras clave Neoplasias de la próstata; comunicación en salud; educación en salud; toma de decisiones. 